\title{
About Possibility of Development Synergetic Processes in Semiconductors of Type $\mathrm{A}^{\mathrm{III}} \mathrm{B}^{\mathrm{V}}$
}

\author{
Ada Leyderman ${ }^{1}$, Amin Saidov ${ }^{1}$, Muslim Khashaev ${ }^{1}$ \& Utkur Rahmonov ${ }^{1}$ \\ ${ }^{1}$ Physic-Technical Institute NPO "Physic-Sun", Academy of Sciences of Uzbekistan, Uzbekistan \\ Correspondence: Ada Leyderman, 100084, 2-B Badamzar Yuli Str., Tashkent, Uzbekistan. E-mail: \\ khashaev@mail.ru
}

Received: November 5, 2012 Accepted: December 10, 2012 Online Published: January 28, 2013

doi:10.5539/jmsr.v2n2p14

URL: http://dx.doi.org/10.5539/jmsr.v2n2p14

Paper is made under grants $\Phi 2-A-0-97004$ and $\Phi 2-A-0-43917$

\begin{abstract}
Possibility of development of processes of selforganization in the semiconductors of type $\mathrm{A}^{\mathrm{III}} \mathrm{B}^{\mathrm{V}}$ grown up by Chohralsky method in which there are no single vacancies because all of them are combined in complexes of type shallow donor + vacancy has been shown. At small homogeneous heating of such samples these complexes break up and periodic distribution of vacancies along the sample appearances. Thus, internal isotype potential barriers are formed. Because of division of created by action of temperature the non-equilibrium carriers by these barriers synergetic current and (or) voltage generate. These theoretical ideas are confirmed by experiments on gallium arsenide doped by tellurium and gallium arsenide doped by tin.
\end{abstract}

Keywords: semiconductors with deep impurities, periodical distribution of vacancies, synergetic current and voltage

\section{Introduction}

Synergetic as independent part of science was shaped in the middle of XX century and as it is will be known from the beginning involved different fields of human knowledge-biology (Turing, 1952), chemistry (Zhabatinskiy, 1974) and at last physics. Due to Prigozhin's works (Glansdorff \& Prigogine, 1971; Nicolis \& Prigogine, 1977) thermodynamic fundamentals of development of processes of self-organization in any open system have been formulated. At present there are many papers and even monographers devoted synergetic processes in semiconductor (Haken, 1978; Scholl, 1987). However there is a special field of physics of semiconductors - physics of semiconductors with deep impurities where these phenomena are researched not enough.

In (Leiderman \& Karageorgy-Alkalaev, 1987; Karageorgy-Alkalaev \& Leiderman, 1987; Leiderman, 2004, 2008) it has been shown theoretically that for development of processes of self-organization in semiconductors with deep impurities it is not enough simple change of recombination rate of non-equilibrium carriers. For that it is necessary to change of numbers of effectively working recombination centers. When their number begins to decrease with the increase of excitation's level inhibition of recombination begins and as a result periodic distribution (not simple spatial one) of concentration of non-equilibrium carriers is formed. It is accompanied by appearance of internal electric field and corresponding potential barriers. At the homogeneous photoexcitation division of free carriers by these barriers causes generation of synergetic current and (or) voltage. The purpose of the given paper is to show the possibility of development synergetic processes in semiconductors of type $A^{\mathrm{III}} \mathrm{B}^{\mathrm{V}}$.

\section{Theoretical Discussion of Possibility of Appearance of Periodic Distribution of Vacancies on the Conditions of Temperature Excitation}

So to have begun synergetic processes it is necessary appearance of periodic distribution of metastable recombination complexes and as consequence of periodic distribution of non-equilibrium carriers. Apparently the similar situation exists in the materials of type $\mathrm{A}^{\mathrm{III}} \mathrm{B}^{\mathrm{V}}$ where possibility of appearance of such periodic distribution is put already in process of their growth by Chohralsky method. 
Now it is well-known that in n-type semiconductors $\mathrm{A}^{\mathrm{III}} \mathrm{B}^{\mathrm{V}}$ grown by Chohralsky method there are practically no free vacancies (see for example Hwang, 1969; Bulyarskiy \& Fistul, 1997). It is caused by that cationic vacancy in compounds $\mathrm{A}^{\mathrm{III}} \mathrm{B}^{\mathrm{V}}$ is rather mobile and being itself as a matter of fact an acceptor is easily related under the influence of a Coulomb interaction to the shallow donor, forming a neutral donor-acceptor complex. In particular in the n-type gallium arsenide doped by tellurium it will be a complex of type vacancy of gallium + donor atom of tellurium. In the case when in the material concentration of shallow donors is more than vacancies' concentration free vacancies will not be. Really it completely proves by researches of paramagnetic resonance as signal EPR which can be related to vacancies, is observed only in p-type GaAs (Williams, 1968). In the given paper attempt of research of processes appearing in such semiconductors under the influence of temperature is made.

Let's write down the equation describing dynamics of change of vacancies' concentrations in such semiconductor under the influence of temperature:

$$
\frac{\partial V}{\partial t}=D_{V} \frac{\partial^{2} V}{\partial x^{2}}+V(Q)-U_{V}+K_{2}(Q) N_{D V}-K_{1}(Q) N_{D V} .
$$

Here the first term on the right part describes diffusion of vacancies ( $D_{V}$ - diffusion constant of vacancies), V(Q) - concentration of the free vacancies which are appeared in unit of time in unit of volume under the influence of temperature, $U_{v} \approx V / \tau_{v}$ - recombination rate of vacancies, $\tau_{v}$ - lifetime of the free vacancies, $N_{D V}$ - concentration of complexes of type shallow donor + vacancy. Term $K_{2}(Q)\left(N_{D V}\right)$ describes process of decay of these complexes under the influence of temperature, and term $K_{I}(Q)\left(N_{D V}\right)$ describes formation of new complexes when formed again vacancies united due to Coulomb interaction with the free shallow donors. It is clearly that $N_{D \sim} \sim V$ as each such complex contains one shallow donor and one vacancy.

If to suppose that concentration of formed again vacancies is very small first two terms in the Equation (1) can be neglected and considering direct proportional dependence between concentration of complexes $N_{D V}$ and vacancies' concentration $V$ on stationary conditions $\left(\frac{\partial V}{\partial t}=0\right)$ it is possible definitively to write down:

$$
D_{V} \frac{\partial^{2} V}{\partial x^{2}}+K_{2}(Q) V-K_{1}(Q) V=0 .
$$

If in the Equation (2) second term corresponding to decay of complexes of type shallow donor + vacancy under the influence of thermal energy is less than the third term describing appearance of such complexes as a result of Coulomb interaction it will be as:

$$
D_{V} \frac{\partial^{2} V}{\partial x^{2}}-K_{1}(Q) V=0
$$

The solution of this equation on any boundary conditions will have exponential character and we will obtain the monotonous distribution of vacancies.

But if decay of the complexes occurs more intensively than their formation, i.e. $K_{2}(Q)>K_{I}(Q)$, Equation (2) can be rewritten down as:

$$
D_{V} \frac{\partial^{2} V}{\partial x^{2}}+K_{2}(Q) V=0
$$

i.e. distribution of vacancies in the researched material is described by the equation of harmonious oscillators. Its solution looks like:

$$
V=C_{1} \cos (\omega \cdot x)+C_{2} \sin (\omega \cdot x),
$$

where $C_{1}$ and $C_{2}$ - the integration constants determined by the boundary conditions. In particular they can be such: $V=V(0)$ at $x=0 ; \quad V=V(d)$ at $x=d$ or $\quad V=V(0), \frac{d V}{d x} \downarrow_{x=0}$ at $x=0$.

The frequency of resulting distribution is described by the expression:

$$
\omega^{2}=\frac{K_{2}(Q)}{D_{V}}
$$


i.e. it depends immediately on intensity of thermal action. Since the coefficient of decay of complexes $K_{2}(Q)$ will increase at increasing temperature frequency of vacancies' distribution will also increase.

The obtained solution has periodic character. It can be also written down as:

$$
V=A \sin (\omega x+\alpha)
$$

where amplitude $A=\sqrt{C_{1}^{2}+C_{2}^{2}}$ and phase $\operatorname{tg} \alpha=-\frac{C_{2}}{C_{1}}$.

Expression (7) can be also rewritten as:

$$
V=V_{0}+V^{*} \sin (\omega \cdot x),
$$

where $V_{0}$ - the average concentration of vacancies, $V^{*}$ - the amplitude of their change.

The periodic nature of the obtained spatial distribution clearly indicates the beginning of the processes of selforganization in the considered system.

From expression (8) it is clearly seen that character of appearing spatial distribution of vacancies' concentration depends on the frequency and the length of the sample. For example if $\omega \cdot d<<1, \sin \omega \cdot x \approx \omega \cdot x$ and $V \approx V_{0}+V^{*} \omega \cdot x ;$ as $V^{*}<V_{0}, V \approx V_{0}$, i.e. distribution of vacancies' concentration practically homogenous. But if $\omega \cdot d=\pi / 2 ; \pi ; 3 \pi / 2 ; 2 \pi$, depending on the relation between frequency and length of the sample distributions look like shown in Figure 1.

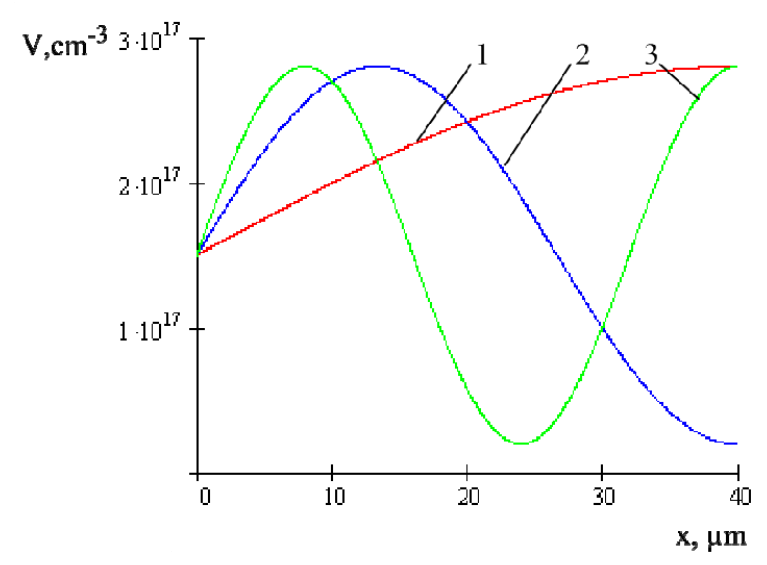

Figure 1. Approximated distribution of vacancy concentration along the sample, according to the Equation (8); The curve $1-\omega \cdot d=\pi / 2$, the curve $2-\omega \cdot d=3 \pi / 2$, the curve $3-\omega \cdot d=5 \pi / 2$

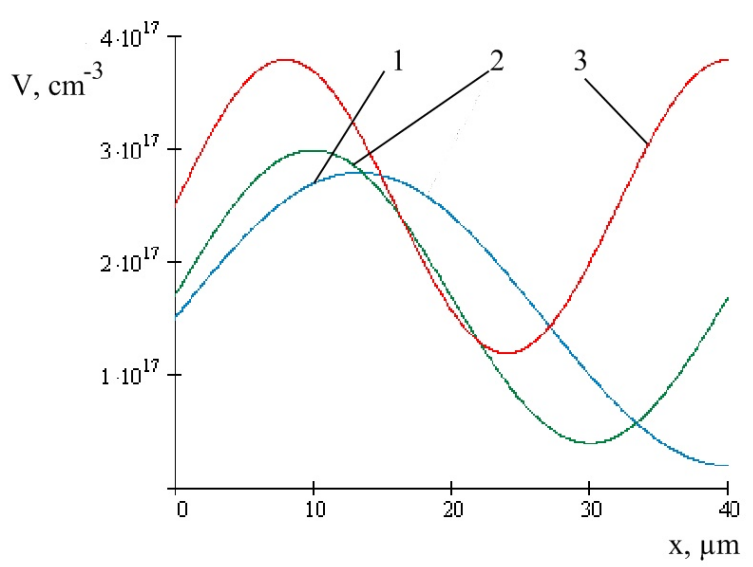

Figure 2. Approximated change of distribution of vacancy concentration at increase of temperature for the curve 2 of Figure 1 
It is known that specific heat capacity of gallium arsenide $c=339 \mathrm{j} /(\mathrm{kg} \cdot \mathrm{K})$. It is possible to estimate thermal energy falling to one atom of gallium arsenide under the formula:

$$
\frac{Q}{N}=\frac{c m \Delta T N_{A}}{m M}=\frac{c \Delta T N_{A}}{M},
$$

where $N$-total number of gallium arsenide atoms, $M$-the molar mass of gallium arsenide, $N_{A}$-Avogadro constant. Calculation shows that the energy falling to one atom at heating $1{ }^{\circ} \mathrm{C}$ is equal $5.1 \times 10^{-4} \mathrm{eV}$. At heating $100{ }^{\circ} \mathrm{C}$ it will be already $5.1 \times 10^{-2} \mathrm{eV}$, and at $150^{\circ} \mathrm{C}$ it is $7.6 \times 10^{-2} \mathrm{eV}$.

Thermal energy falling $1 \mathrm{~cm}^{3}$ is equal $\frac{Q}{V}=c \rho \Delta T=1.14 \cdot 10^{13} \frac{\mathrm{eV}}{\mathrm{cm}^{3}}$. We divide that on width of forbidden gap $E_{g}=$ $1.41 \mathrm{eV}$ and we obtain quantity of carriers which can be generated in conduction band from $1 \mathrm{~cm}^{3}$ at heating $1{ }^{\circ} \mathrm{C}$ : $\mathrm{n}=8.14 \times 10^{12} \mathrm{~cm}^{-3}$. At heating $100^{\circ} \mathrm{C}$ this quantity increases to $\mathrm{n}=8.14 \times 10^{14} \mathrm{~cm}^{-3}$. It is necessary to note that the given calculation is rather approximated since it does not take into account thermal energy's losses on oscillations of the lattice and supposed that all thermal energy is spent on transmission of appearing free carriers in conduction band.

Really the mechanism of such processes is following: heating creates phonons which sum with very small probability in the necessary quantity of the phonons operating simultaneously on an electron in valence band. If the energy of all these phonons is enough for transmission (i.e. it is equal or more than $E_{g}$ ) the electron transfers in conduction band. These typically non-linear optical processes are very improbable. Naturally the more the temperature the more electrons in conduction band will be. This electron's concentration (initial one) is determining by the known expression (see for example Anselm, 1978):

$$
n=\frac{\left(2 \pi \sqrt{m_{n} m_{p}} k T\right)^{3 / 2}}{4 \pi^{3} \hbar^{3}} e^{-\frac{E_{G}}{2 k T}},
$$

where $m_{n}, m_{p}$ - effective masses of electrons and holes, $E_{g}$-forbidden gap width. Then it is easy to estimate that concentration of free carriers at heating $1{ }^{\circ} \mathrm{C}$ changes to $\Delta n=10^{5} \mathrm{~cm}^{-3}$ and at heating $100{ }^{\circ} \mathrm{C}$ changes to $\Delta n=10^{9} \mathrm{~cm}^{-3}$. Apparently the discrepancy in concentration estimates makes five orders. But it is no wonder as in our initiating estimate it was supposed that all thermal energy is spent for making of the free carriers in conduction band.

Certainly energy of the order $0.1 \mathrm{eV}$ is insignificant and it can be insufficient for decay of complexes. However it is necessary to note that at heating even $1{ }^{\circ} \mathrm{C}$ process of excitation of the free carriers takes place; it inevitably causes their partial recombination and only then the new equilibrium state becomes. Thus in each act of recombination the energy equal approximately to half of width of forbidden gap is emitted and this energy can be enough for decay of the complexes, i.e. recombination - stimulated processes of changes of impurities and defects will take place. For the first time these processes were predicted for gallium arsenide by Gold and Kimerling (Gold \& Weinberg, 1964; Kimerling, 1978) and then they were researched for the recombination in semiconductors with the deep impurities (Leiderman \& Karageorgy-Alkalaev, 1987; Karageorgy-Alkalaev \& Leiderman, 1987). In this case coefficient $K_{2}$ will be function not only thermal energy but also injection level and then frequency of the spatial distribution of vacancies becomes function of injection's level as the result of recombination - stimulated processes.

Presence of periodic distribution of vacancies unequivocally testifies to appearance of internal electric field of Dember type:

$$
E_{D}=-\frac{k T}{q} \cdot \frac{b-1}{b+1} \cdot\left(\frac{d p}{d x}\right) \cdot p^{-1}
$$

where $k$ - Boltzmann constant, $q$ - the elemental charge, $b=\mu_{n} / \mu_{p}$ - the relation of mobility of electrons and that of holes, $p$ - concentration of the free holes.

Hence free carriers appearing at heating will be divided by that potential barrier and generate the voltage:

$$
V_{D} \equiv \int_{0}^{d} E_{D} d x=-\frac{k T}{q} \cdot \frac{b-1}{b+1} \cdot \ln \frac{p(d)}{p(0)},
$$

where $p(0)$ and $p(d)$ - concentrations of the free carriers on the edges of the sample. 
Taking into account (7) it is possible expression (12) to write down as

$$
V_{D}=-\frac{k T}{q} \cdot \frac{b-1}{b+1} \cdot \ln \frac{V_{0}+V^{*} \sin \omega \cdot d}{V_{0}+\left.V^{*}[\sin \omega \cdot x]\right|_{x=0}},
$$

where $d$ - length of the sample.

Thus in researched material can exists periodic distribution of the vacancies; its appearance depends on temperature. Hence isotype potential barrier of $n-n^{+}$-type exists or on the other hand there is an internal electric field. In this case at appearance of the free carriers in the material, in particular at influence of thermal energy created at the homogeneous heating their division by this barrier should be, i.e. it is possible appearance of thermo-stimulated currents and voltages. To verify this idea we researched samples of n-type gallium arsenide obtained by Chohralsky method doped by tellurium and similar samples doped tin.

\section{Experimental Researches of Thermo-Stimulated Processes in Gallium Arsenide Doped by Tellurium and Gallium Arsenide Doped by Tin}

From the plate of gallium arsenide doped by tellurium with initial electron concentration $n_{n}=3 \cdot 10^{18} \mathrm{~cm}^{-3} 30$ $\mathrm{mm}$ in diameter, 480 microns thick have been made samples $7 \mathrm{~mm}$ wide and $13 \mathrm{~mm}$ long. Simple ohmic contacts of silver have been attached to these samples: on the one side - continuous contact and on the other - contact in the form of a strip. The results of measuring the temperature-current dependence and temperature-voltage dependence are presented in Figure $3 a$ and $3 b$.

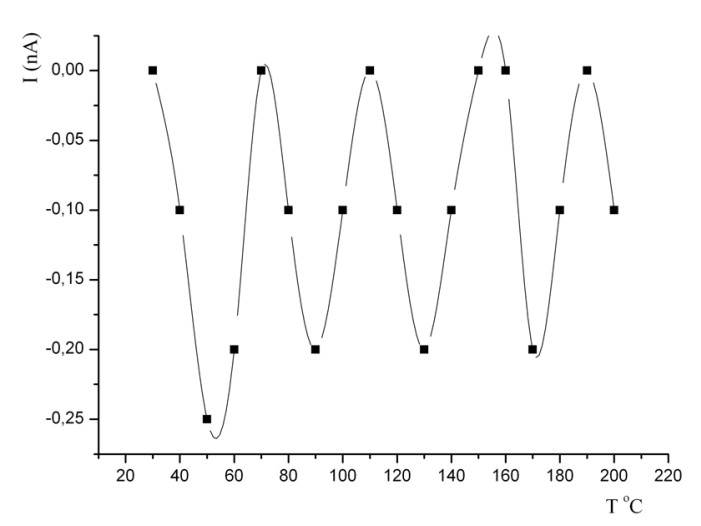

a)

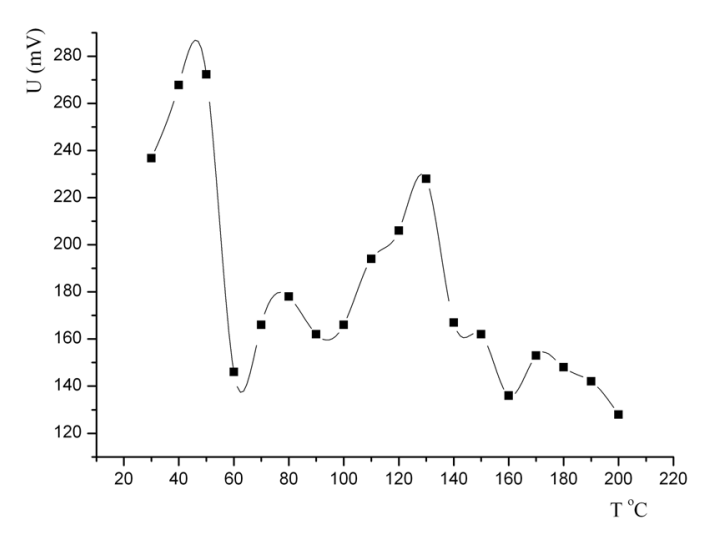

b)

Figure 3. Temperature-current dependence (a) and temperature-voltage dependence (b) for n-type sample GaAs $<\mathrm{Te}>$ with ohmic contacts

If in the researched material there is the periodic distribution of vacancies and creating by that isotype $n-n{ }^{+}$ potential barriers their existence should affect current-voltage characteristics. For the purpose of verify of this idea we have measured current-voltage characteristics of the given structure with simple ohmic contacts. Results of measuring current-voltage characteristics at different temperatures are shown in Figure 4. It is seen that at the room temperature current-voltage characteristic is practically ohmic that corresponds to standard representations about structure with ohmic contacts. However with temperature increase there are diversions which are increase at temperature increase. The greatest diversions are observed at temperature $150{ }^{\circ} \mathrm{C}$. Thus observed current-voltage characteristics also testify to presence in researched material of any rectifying barrier by which division of non-equilibrium carriers created by action of temperature takes place.

Further n-type samples gallium arsenide doped by tin obtained by Chohralsky method with initial electron concentration $n_{n}=1.1 \div 3.4 \cdot 10^{17} \mathrm{~cm}^{-3}$ were researched. From the plate of gallium arsenide doped by tin $30 \mathrm{~mm}$ in diameter samples $6 \mathrm{~mm}$ wide and $5 \mathrm{~mm}$ long have been made. Simple ohmic contacts of silver have been attached to these samples: on the one side - continuous contact and on the other - contact in the form of a strip. The results of measuring the temperature-current dependence and temperature-voltage dependence are presented in Figures $5 \mathrm{a}$ and $5 \mathrm{~b}$. The estimate of the maximal quantity of the obtained voltage (13) at temperature $300{ }^{\circ} \mathrm{C}$ 
and relation vacancy concentrations $V_{0} / V^{*}=1.5$ gives $1.9 \mathrm{mV}$, that is in the good consent with the maximal voltage of Figure $5 \mathrm{~b}$.

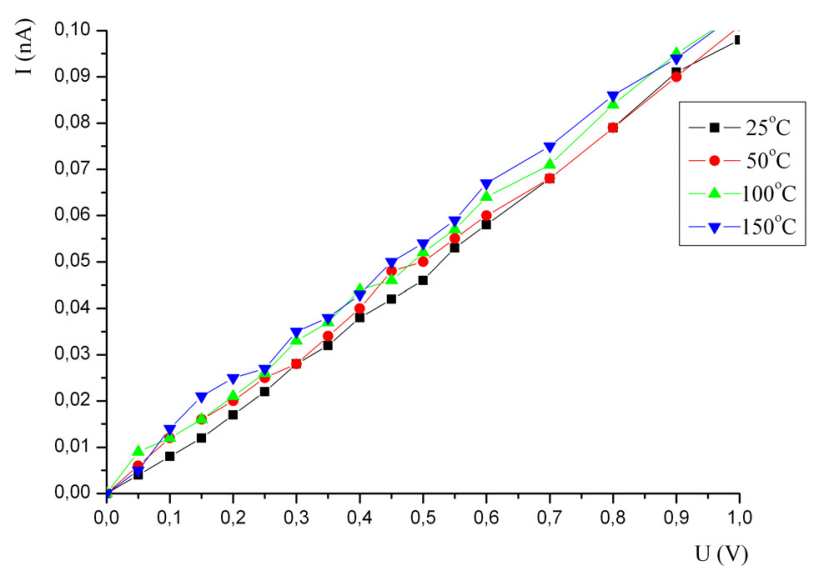

Figure 4. Current-voltage characteristics of $\mathrm{R}_{\mathrm{Ohm}}-\mathrm{n}-\mathrm{GaAs}-<\mathrm{Te}>-\mathrm{R}_{\mathrm{Ohm}}$ structure with simple ohmic contacts at different temperatures

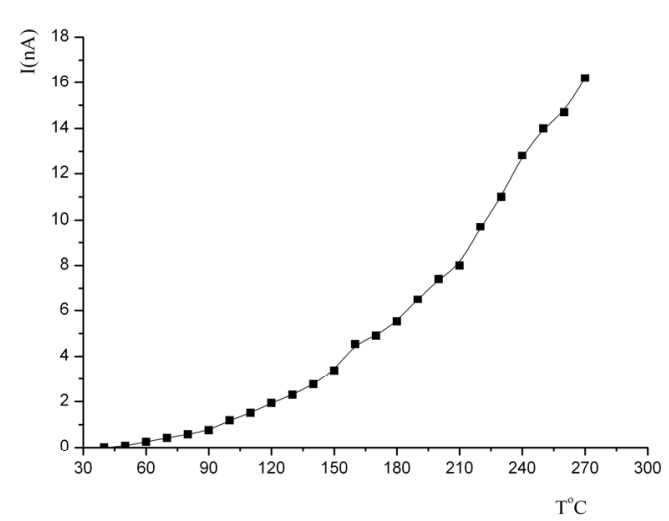

a)

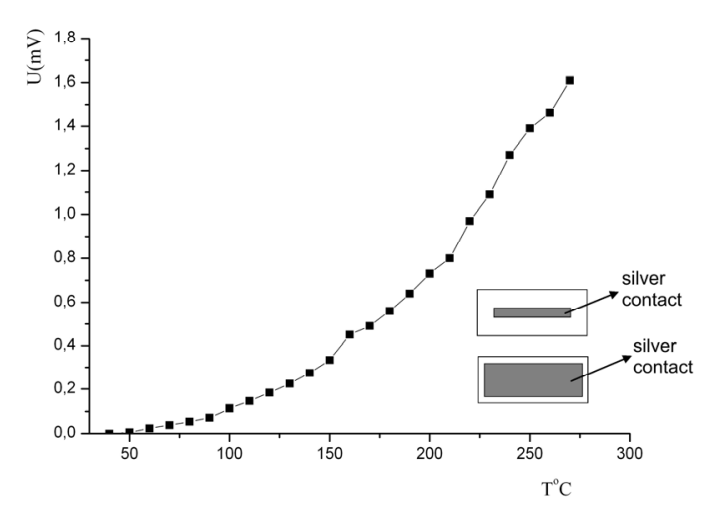

b)

Figure 5. Temperature-current dependence (a) and temperature-voltage dependence (b) for n-type sample GaAs $<\mathrm{Sn}>$ with ohmic contacts (schematic view of the sample is shown on the right in Figure $3 \mathrm{~b}$ )

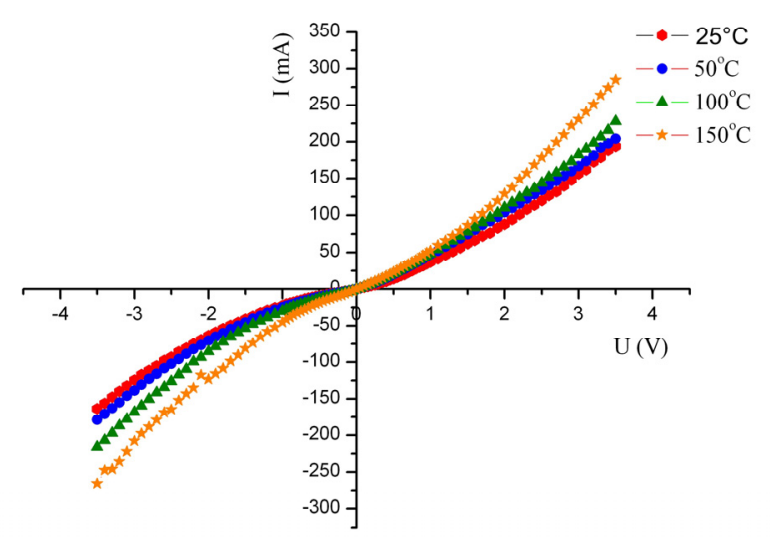

Figure 6. Current-voltage characteristics of $\mathrm{R}_{\mathrm{Ohm}}-\mathrm{n}-\mathrm{GaAs}-<\mathrm{Sn}>-\mathrm{R}_{\mathrm{Ohm}}$ structure with simple ohmic contacts 
Thus in the researched material can exist a periodic distribution of the vacancies which depends on temperature. Hence isotype potential barriers of $n-n^{+}$-type can exist. In this case at appearance in the material of free carriers in particular because of thermal energy created at the homogeneous heating their division by this barrier should be. It explains appearance of thermo-stimulated currents and voltages. Results of measuring current-voltage characteristics are shown in Figure 6. Current-voltage characteristics both Figure 4 and Figure 6 testify unequivocally to presence in the researched material of any rectifying potential barrier on which there is division of the free carriers created by heating. It is clearly seen that nonlinearity of current-voltage characteristics increase at increase of temperature.

Thus these researches show possibility of thermo-stimulated voltaic phenomena in the n-type gallium arsenide doped by tellurium and n-type gallium arsenide doped by tin.

\section{Conclusion}

Thus possibility of development of processes of selforganization in semiconductors $\mathrm{A}^{\mathrm{III}} \mathrm{B}^{\mathrm{V}}$ of $\mathrm{n}$-type conductivity grown up by Chohralsky method has been shown. In such semiconductors at the beginning there are no free vacancies because of they are combined in defect impurity complexes of type vacancy + shallow donor. Under influence of homogeneous heating these complexes break up and periodic distribution of vacancies along the sample appears, i.e. internal isotype potential barriers appear. Division of created by heating non-equilibrium carriers by these potential barriers is the cause of generation of synergetic current and (or) voltage. These theoretical ideas are confirmed experimentally on sample with the simple ohmic contacts fabricated from of $\mathrm{n}-\mathrm{GaAs}<\mathrm{Te}>$ and $\mathrm{n}-\mathrm{GaAs}<\mathrm{Sn}>$. It is necessary to note that apparently the same phenomena can be also observed in n-type gallium arsenide doped by 4 group's impurities, and also in GaP. In particular in the last complexes of type $\mathrm{V}_{\mathrm{Ga}} \mathrm{S}$ (vacancy of gallium + sulphur) are.

It would note that last years a number of papers in which possibility of development of thermovoltaic effects in different materials because of appearance non-uniform distribution of impurities has been researched both theoretically and experimentally. In particular it can be shown papers devoted researches in sulphide of samarium (Kazanin, Kaminski, \& Solov'ev, 2000; Kaminski, Golubkov, \& Vasil'ev, 2002; Kaminski \& Kazanin, 2009).

It is necessary to underline that in case of appearance of periodic dislocation of vacancies when they play role of recombination centers theory of all excitation - photoexcitation, injection, temperature should be made again.

\section{References}

Anselm, A. I. (1978). Vvedenie v phiziku poluprovodnikov. Moscow, Nauka.

Bulyarskiy, S. V., \& Fistul, V. I. (1997). Termodinamika $i$ kinetika vzaimodeystvuyushih defektov $v$ poluprovodnikah. Moscow, Nauka, Fizmatlit.

Glansdorff, P., \& Prigogine, I. (1971). Thermodynamic theory of structure, stability and fluctuations. New York, NY: Wiley-Intercinc.

Gold, E. D., \& Weinberg, L. E. (1964). Permanent degradation of GaAs tunnel diodes. Solid-State Electron., 7(3), 811-821. http://dx.doi.org/10.1016/0038-1101(64)90133-9

Haken, H. (1978). Synergetics. New York, NY: Springer Verlag Berlin Heidelberg.

Hwang, C. J. (1969). Optical Properties of n-Type GaAs. II. Formation of Efficient Hole Traps during Annealing in Te-Doped GaAs. J. Appl. Phys., 40, 4584-4592. http://dx.doi.org/10.1063/1.1657236

Kaminski, V. V., Golubkov, A. V., \& Vasil'ev, L. N. (2002). Defect samarium ions and electromotive-force generation in SmS. Physics of the Solid State, 44(8), 1574-1578. http://dx.doi.org/10.1134/1.1501362

Kaminski, V. V., \& Kazanin, M. M. (2009). Thermovoltaic effect in polycrystalline samarium sulphide. Technical Physics Letters, 35(11), 981-984. http://dx.doi.org/10.1134/S1063785009110030

Karageorgy-Alkalaev, P. M., \& Leiderman, A. Yu. (1987). The synergetic phenomena in photoexcited semiconductors. Phys. Stat. Sol. (A), 100(1), 221-231. http://dx.doi.org/10.1002/pssa.2211000125

Kazanin, M. M., Kaminski, V. V., \& Solov'ev, S. M. (2000). Anomalous thermal electromotive force in samarium monosulfide. Technical Physics, 45(5), 659-661. http://dx.doi.org/10.1134/1.1259698

Kimerling, J. (1978). Recombination enhanced defect reactions. Solid-State Electron., 21(11), 1391-1401. http://dx.doi.org/10.1016/0038-1101(78)90215-0

Leiderman, A. Yu., \& Karageorgy-Alkalaev, P. M. (1987). Cryst. Latt. Def. and Amorph. Mat., 13(3-4), 323-327. 
Leiderman, A. Yu. (2004). About possibility space-waves and time-waves of concentration's non-equilibrium carriers in semiconductors with deep impurities. 8th International Conference "Solid State Physics", pp. 298-303, August 23-26, Almaty, Kazakhstan.

Leiderman, A. Yu., Stel'makh, V. G., \& Sadykov, M. (2008). Recombination in semiconductors with deep impurities. Applied Solar Energy, 44(4), 276-280. http://dx.doi.org/10.3103/S0003701X08040105

Nicolis, G., \& Prigogine, I. (1977). Self-organization in non-equilibrium systems. New York, NY: Wiley.

Turing, A. M. Phill. (1952). Trans. Roy. Soc. (237, 37-72). London: Der. B.

Scholl, E. (1987). Nonequilibrium Phase Transitions in Semiconductors. Self-Organization Induced by Generation and Recombination Processes. New York, London, Paris, Tokyo: Springer-Verlag Berlin Heidelberg

Williams, E. W. (1968). Evidence for Self-Activated Luminescence in GaAs: The Gallium Vacancy-Donor Center. Phys. Rev., 168, 922-932. http://dx.doi.org/10.1103/PhysRev.168.922

Zhabatinskiy, A. M. (1974). Konzentrazionnie avtokolebaniya. Moscow: Nauka. 